\title{
De novo Biosynthesis of Tyrosol Acetate and Hydroxytyrosol Acetate from Glucose in Engineered Escherichia Coli
}

Daoyi Guo ( $\sim$ ggdy3478@163.com )

Gannan Normal University

Xiao Fu

Gannan Normal University

Yue Sun

Gannan Normal University

Xun Li

Gannan Normal University

Hong Pan

Gannan Normal University

Research

Keywords: Metabolic engineering, Escherichia coli, Tyrosol acetate, Hydroxytyrosol acetate,

Posted Date: March 11th, 2021

DOI: https://doi.org/10.21203/rs.3.rs-287007/v1

License: (c) (1) This work is licensed under a Creative Commons Attribution 4.0 International License. Read Full License 


\section{Abstract}

Background: Tyrosol and hydroxytyrosol derived from virgin olive oil and olives extract, have wide applications both as functional food components and as nutraceuticals. However, they have low bioavailability due to their low absorption and high metabolism in human liver and small intestine. Acetylation of tyrosol and hydroxytyrosol can effectively improve their bioavailability and thus increase their potential use in the food and cosmeceutical industries. There is no report on the bioproductin of tyrosol acetate and hydroxytyrosol acetate so far. Thus, it is of great significance to develop microbial cell factories for achieving tyrosol acetate or hydroxytyrosol acetate biosynthesis.

Results: In this study, two de novo biosynthetic pathways for the production of tyrosol acetate and hydroxytyrosol acetate were constructed in Escherichia coli. First, an engineered E. coli that allows production of tyrosol from simple carbon sources was established. Four aldehyde reductases were compared, and it was found that yeaE is the best aldehyde reductase for tyrosol accumulation. Subsequently, the pathway was extended for tyrosol acetate production by further overexpression of alcohol acetyltransferase ATF1 for the conversion of tyrosol to tyrosol acetate. Finally, the pathway was further extended for hydroxytyrosol acetate production by overexpression of 4-hydroxyphenylacetate 3hydroxylase HpaBC.

Conclusion: We have successfully established the artificial biosynthetic pathway of tyrosol acetate and hydroxytyrosol acetate from fermentable sugars and demonstrated for the first time the direct fermentative production of tyrosol acetate and hydroxytyrosol acetate from glucose in engineered $E$. coli

\section{Background}

Plant phenolic compounds have antioxidant and other beneficial biological activities, so they have wide applications both as functional food components and as nutraceuticals [1]. Among these phenolic compounds, tyrosol and hydroxytyrosol derived from virgin olive oil and olives extract, have attracted extensive attention[2]. Tyrosol and hydroxytyrosol are widely recognized as an antioxidant, antiinflammatory molecule that inhibits platelet aggregation and plays a protective role in the heart $[3,4]$. However, they have low bioavailability due to their low absorption and high metabolism in human liver and small intestine[5].

Acetylation of phenolic compounds has been reported to enhance lipophilicity as well as absorption and cell permeability, thus improving their bioavailability[6]. Tyrosol acetate derivatives showed better antimicrobial and antileismaniac activities than tyrosol. This effect can be attributed to its increased lipophilicity $[7,8]$. Tyrosol acetate also showed a higher cytotoxic effect on cancer lines than tyrosol[9]. On the other hand, hydroxytyrosol acylation can improve antioxidant activity, decrease tumor necrosis factor (TNF) and interleukin (IL) 1B plasma levels, decrease IL1B and chemokine ligand 2 levels of adipose tissue and showed greater anti-inflammatory effects than hydroxytyrosol[10-12]. This acetylation can be explored by reaction with acid chlorides or acid anhydrides, but these chemical routes do not meet the 
requirements necessary for food applications. Several enzymatic methods have been reported for the preparation of tyrosol acetate or hydroxytyrosol acetate[13]. However, there are some drawbacks by using enzymes in bioprocesses such as the need of expensive enzymes and substrates. Thus, it is of great significance to develop microbial cell factories for achieving tyrosol acetate or hydroxytyrosol acetate biosynthesis. In this study, two de novo biosynthetic pathways for the production of tyrosol acetate and hydroxytyrosol acetate were constructed in E. coli (Fig. 1).

\section{Methods}

\section{Plasmid and strains}

Plasmid pDG11 for expression of the feedback resistant mutant of 3-deoxy-D-arabinoheptulosonate-7phosphate synthase AroG ${ }^{\mathrm{fbr}}$ (D146N) was constructed in our previous study[17]. The feedback resistant mutant of chorismate mutase/prephenate dehydrogenase tyrA ${ }^{\mathrm{fbr}}$ was synthesized by Genewiz Biotech Co. Ltd., amplified by PCR using primers tyrA ${ }^{\mathrm{fbr}}{ }_{-\mathrm{Xbal}}$ and tyrA ${ }^{\mathrm{fbr}}$-Spel-Sall. The Xbal-Sall fragment of tyr $\mathrm{A}^{\mathrm{fbr}}$ was inserted into Spel and Sall sites of pDG11 to give pDG20. The ketoacid decarboxylase KDC gene were amplified by PCR from from S. cerevisiae genomic DNA using primers KDC-Xbal and KDC-NhelBamHI, and inserted into pBBRMCS1 via Xbal and BamHI to to give pDG21. The Xbal-Sall fragment of AroG $^{\mathrm{fbr}}$ and tyrA ${ }^{\mathrm{fbr}}$ from pDG20 was inserted into Nhel and Xhol sites of pDG21 to give pDG22.

Aldehyde reductases (gahK, dkgB, yeaE and gyhD) were individually amplified by PCR from E. coli genomic DNA using primers gahK - Xbal/gahK - Nhel - BamHI, dkgB - Xbal/ dkgB - Nhel - BamH, yeaE Xbal/ yeaE - Nhel - BamHI and gyhD-Xbal / gyhD - Nhel - BamHI, and ligated into pET28a(+) via Xbal and BamHI to yield plasmid pDG23, pDG24, pDG25 and pDG26. Alcohol acetyltransferase ATF1 gene was amplified by PCR from S. cerevisiae genomic DNA using primers ATF1-Xbal and ATF1-Nhel-Sacl, and inserted into pET28a(+) to give pDG27. The Xbal-Xhol fragment of ATF1 from pDG27 was inserted into Nhel and Xhol sites of pDG25 to give pDG28. 4-Hydroxyphenylacetate 3-hydroxylase HpaBC was amplified by PCR from E. coligenome using primers HpaBC-Xbal and $\mathrm{HpaBC}-X$ hol, and inserted into Nhel and Xhol sites of pDG28 to give pDG29. The strains, primers and plasmids used in this study are summarized in Table 1 and Table 2. 
Table 1

Primers used in this study.

\begin{tabular}{|c|c|}
\hline Primer name & Sequence $\left(5^{\prime}-3^{\prime}\right)$ \\
\hline tyrA ${ }^{\text {fbr-Xbal }}$ & GTATCTAGAAAGAGGAGATATAATGGTTGCTGAATTGACCGCAT \\
\hline tyrA ${ }^{\text {fbr-Spel-Sall }}$ & ATATGTCGACACTAGTTTACTGGCGATTGTCATTCGCC \\
\hline KDC-Xbal & atctctagatttaagaaggagatataatggcacctgttacaattgaaaagttc \\
\hline KDC-Nhel-BamHI & tctggatccgctagcctattttttatttcttttaagtgccgctg \\
\hline gahK - Xbal & ATCTCTAGATTTAAGAAGGAGATATAATGAAGATCAAAGCTGTTGGTGCATAT \\
\hline $\begin{array}{l}\text { gahK - Nhel - } \\
\text { BamHI }\end{array}$ & TCTGGATCCGCTAGCTCAGTCTGTTAGTGTGCGATTATCGATAA \\
\hline $\mathrm{dkgB}-\mathrm{Xbal}$ & ATCTCTAGATTTAAGAAGGAGATATAATGGCTATCCCTGCATTTGGTTTAG \\
\hline $\begin{array}{l}\text { dkgB - Nhel - } \\
\text { BamH }\end{array}$ & TCTGGATCCGCTAGCTTAATCCCATTCAGGAGCCAGACC \\
\hline yeaE - Xbal & ATCTCTAGATTTAAGAAGGAGATATAATGCAACAAAAAATGATTCAATTTAGTGG \\
\hline $\begin{array}{l}\text { yeaE - Nhel - } \\
\text { BamH }\end{array}$ & TCTGGATCCGCTAGCTCACACCATATCCAGCGCAGTTT \\
\hline gyhD-Xbal & ATCTCTAGATTTAAGAAGGAGATATAATGAACAACTTTAATCTGCACACCCC \\
\hline $\begin{array}{l}\text { gyhD - Nhel - } \\
\text { BamHI }\end{array}$ & TCTGGATCCGCTAGCTTAGCGGGCGGCTTCGTATATAC \\
\hline ATF1-Xbal & GGATCTAGAAACTTTAAGAAGGAGATATAATGAATGAAATCGATGAGAAAAATCAGG \\
\hline ATF1-Nhel-Sacl & GATGAGCTCACTAGTCTAAGGGCCTAAAAGGAGAGCTTTGTAA \\
\hline HpaBC-Xbal & tagtctagatttaagaaggagatataatgaaaccagaagatttccgcg \\
\hline HpaBC-Xhol & aacgctcgagttaaatcgcagcttccatttcca \\
\hline
\end{tabular}


Table 2

Plasmids and strains used in this study

\begin{tabular}{|lll|}
\hline Plasmids & Properties & Source \\
\hline pDG22 & pBBRMCS1; pT7: KDC, aroG fbr and tyrA ${ }^{\text {fbr }}$ & This study \\
\hline pDG23 & pET28a; pT7: gahK & This study \\
\hline pDG24 & pET28a; pT7: dkgB & This study \\
\hline pDG25 & pET28a; pT7: yeaE & This study \\
\hline pDG26 & pET28a; pT7: gyhD & This study \\
\hline pDG28 & pET28a; pT7: yeaE and ATF1 & This study \\
\hline pDG29 & pET28a; pT7: yeaE, ATF1 and HpaBC & This study \\
\hline Strains & & \\
\hline DG01 & BL21 / pDG 22 & This study \\
\hline DG02 & BL21 / pDG 22/ pDG23 & This study \\
\hline DG03 & BL21 / pDG 22/ pDG24 & This study \\
\hline DG04 & BL21 / pDG 22/ pDG25 & This study \\
\hline DG05 & BL21 / pDG 22/ pDG26 & This study \\
\hline DG06 & BL21 / pDG 22/ pDG28 & This study \\
\hline DG07 & BL21 / pDG 22/ pDG29 & This study \\
\hline
\end{tabular}

\section{Shake Flask Cultures}

Strains were cultured in $100 \mathrm{~mL} \mathrm{M9}$ medium with $2 \%$ glucose at $30^{\circ} \mathrm{C}$ as previously described by Guo[17]. $36 \mathrm{mg} / \mathrm{L}$ chloromycetin and $50 \mathrm{mg} / \mathrm{L}$ kanamycin were added to $\mathrm{M} 9$ medium. When the medium $\mathrm{OD}_{600}$ reached about 0.8 , isopropyl $\beta$-D-thiogalactoside was added with a concentration of $0.1 \mathrm{mM}$.

\section{Analytical Methods}

Cultures samples ( $5 \mathrm{~mL}$ for tyrosol or $10 \mathrm{~mL}$ for tyrosol acetate/hydroxytyrosol acetate) were transferred to $20-\mathrm{mL}$ glass tubes. Glass beads $(0.1 \mathrm{~mm})$ were used for breaking the cells by vigorous vortexing for 5 minutes. Equal volume of ethyl acetate was used to extract fermentation products. After the centrifuge stratification, the upper ethyl acetate phase was withdrawn, evaporated to near dryness, and redissolved in $1.0 \mathrm{~mL}$ of ethyl acetate. A $1 \mu \mathrm{L}$ of the ethyl acetate phase was analysed after splitless injection on an Agilent 7890A GC equipped with an Agilent 5975 MS detector and an Agilent HP-5MS capillary column. 
Helium was used as carrier gas. The temperatures of the injector and detector were $300^{\circ} \mathrm{C}$ and $250^{\circ} \mathrm{C}$, respectively. The following temperature programme was applied: $80^{\circ} \mathrm{C}$ for $2 \mathrm{~min}$, increase of $20^{\circ} \mathrm{C}$ min to $260^{\circ} \mathrm{C}, 260^{\circ} \mathrm{C}$ for $8 \mathrm{~min}$. Benzyl alcohol was used as internal standard for quantitative tyrosol. Methyl benzoate was used as internal standard for quantitative tyrosol acetate and hydroxytyrosol acetate.

\section{Result}

\section{Screening aldehyde reductase for biosynthesis of tyrosol}

Recently, several research groups have reported the biosynthesis of tyrosol in $E$. coli using the intrinsic aldehyde reductase of $E$. coli[14-16]. However, which aldehyde reductase is primarily responsible for catalyzing the reduction of 4-hydroxyphenylacetaldehyde to tyrosol has not been identified. Aldehyde reductase is a superfamily enzyme which catalyze the reduction of a large variety of aldehydes. There are multiple aldehyde reductases in E. coli, such as gahK, yeaE, dkgB and gyhD. In this study, we assembled a tyrosol biosynthetic pathway with various aldehyde reductases in $E$. coli and assessed these aldehyde dehydrogenases for biosynthesis of tyrosol from glucose. The biosynthetic pathway consists of four enzymes: aroG ${ }^{\mathrm{fbr}}$ and $\mathrm{TyrA} \mathrm{fbr}^{\mathrm{fbr}}$ for the efficient overproduction of 4-hydroxyphenylpyruvic acid, ketoacid decarboxylase KDC from S. cerevisiae for the conversion of 4-hydroxyphenylpyruvic acid to 4hydroxyphenylacetaldehyde, aldehyde reductase from $E$. coli for the conversion of 4hydroxyphenylacetaldehyde to tyrosol. The resulting strains DG01-05 were grown in M9 medium with 20 $\mathrm{g} / \mathrm{L}$ of glucose. The fermentation products were extracted by ethyl acetate and analyzed by GC-MS (Fig. 2). The tyrosol yield of the strains with overexpressing different aldehyde reductase were compared with that of the control strain without overexpressing aldehyde reductase. The results showed that yeaE was the most effective aldehyde reductase for biosynthesis of tyrosol, which resulted in an approximately $34 \%$ increase in tyrosol production up to $685 \pm 31.1 \mathrm{mg} / \mathrm{L}$ compared the control strain DG01 (Table 3).

Table 3

Tyrosol production in engineered E. coli strains. All experiments were performed in triplicate and SD is indicated.

\begin{tabular}{|ll|}
\hline Strains & Tyrosol (mg/L) \\
\hline DG01 & $512 \pm 28.3$ \\
\hline DG02 & $558 \pm 21.2$ \\
\hline DG03 & $614 \pm 32.5$ \\
\hline DG04 & $685 \pm 31.1$ \\
\hline DG05 & $631 \pm 22.6$ \\
\hline
\end{tabular}


Besides tyrosol, the small amounts of phenylethanol, phenylethyl acetate and tyrosol acetate were also observed in these recombinant $E$. coli strains, as revealed by GC/MS analysis (Fig. 2). We infer that phenylpyruvate, L-phenylalanine pathway intermediate, can be decarboxylated by 2-keto acid decarboxylase KDC to phenylacetaldehyde, which is subsequently reduced by aldehyde dehydrogenase to phenylethanol. Subsequently, a small amount of phenylethanol and tyrosol were converted to phenylethyl acetate and tyrosol acetate by an intrinsic alcohol acetyltransferase-like enzyme of $E$. coli[17].

\section{Production Tyrosol Acetate From Glucose}

A small amount of tyrosol acetate was produced from tyrosol via an intrinsic alcohol acetyltransferaselike enzyme of E. coli. However, the efficiency is quite low. In order to improve the biosynthesis of tyrosol acetate, strain needs to strengthen the activity of alcohol acetyltransferase. Alcohol acetyltransferase ATF1 from S. cerevisiae has been used for acetylation of a variety of alcohol [18-21]. Therefore, we speculated that the observed promiscuity of the ATF1 can extend also to tyrosol. In this study, a fermentative route for biosynthesis of tyrosol acetate was created by further heterologous expression of ATF1 for the conversion of tyrosol to tyrosol acetate in the tyrosol-producing strain. The fermentation products were extracted by ethyl acetate and analyzed by GC-MS (Fig. 3). The resulting E. coli strain DG06 produced up to $507 \pm 16.9 \mathrm{mg} / \mathrm{L}$ tyrosol acetate within $28 \mathrm{~h}$ (Table 4 ). This proves that the overexpression of ATF1 can effectively enhance the acetylation of tyrosol.

Table 4

Tyrosol acetate and hydroxytyrosol acetate production in engineered

E. coli strains. All experiments were performed in triplicate and SD is indicated.

\begin{tabular}{|lll|}
\hline Strains & Tyrosol acetate(mg/L) & Hydroxytyrosol acetate(mg/L) \\
\hline DG06 & $507 \pm 16.9$ & \\
\hline DG07 & $281 \pm 11.3$ & $225 \pm 9.8$ \\
\hline
\end{tabular}

\section{Production Hydroxytyrosol Acetate From Glucose}

4-Hydroxyphenylacetate 3-hydroxylase $\mathrm{HpaBC}$ is widely distributed in many microorganisms including $E$. coli. HpaBC from E. coli can catalyze the hydroxylation of tyrosol to hydroxytyrosol[22]. Therefore, we hypothesized that the hydroxytyrosol acetate could be biosynthesized by tyrosol-producing $E$. coli through the expression of $\mathrm{HpaBC}$ that catalyzes the hydroxylation of tyrosol to hydroxytyrosol and alcohol acetyltransferase ATF1 that catalyzes the acetylation of hydroxytyrosol to hydroxytyrosol acetate. In this study, the pathway was further extended for hydroxytyrosol acetate production by further overexpression of HpaBC and ATF1 in the tyrosol-producing strain. The resulting E. colis strain DG07 produced up to $225 \pm 9.8 \mathrm{mg} / \mathrm{L}$ hydroxytyrosol acetate within $28 \mathrm{~h}$ (Table 4). This proves that the designed hydroxytyrosol acetate synthetic pathway from glucose was effective in $E$. coli. 


\section{Discussion}

Acetylated tyrosol and hydroxytyrosol can effectively improve their lipophilicity and thus promote their absorption and cell permeability[12, 23, 24]. This acetylation can be accomplished by acid-base catalysis, but these chemical methods do not meet the requirements necessary for food applications. In recent years, a number of pioneering studies have demonstrated the application of lipase for the bioproduction of tyrosol acetate and hydroxytyrosol acetate[13]. Aissa et al. reported that tyrosol acetate was synthesized using immobilized Staphylococcus xylosus lipase through transesterification between ethyl acetate and tyrosol[13]. Alcudia et al. reported that the synthesis of hydroxytyrosol acetate by pancreatic lipase with $86 \%$ yield after $48 \mathrm{~h}$ of reaction[25]. Although enzymes are preferred over chemical catalyst, it is not used widely in industry due to the high cost of enzymes and substrates.

To meet the increasing market demand, engineering microbes to produce tyrosol acetate and hydroxytyrosol acetate from renewable resources represents a promising alternative. In this study, two de novo biosynthetic pathways for the production of tyrosol acetate and hydroxytyrosol acetate were constructed in E coli. First, an engineered $E$. coli that allows production of tyrosol from simple carbon sources was established. Four aldehyde reductase were compared, and it was found that yeaE is the best aldehyde reductase for tyrosol accumulation. Subsequently, the pathway was further extended for tyrosol acetate and hydroxytyrosol acetate production by further overexpression of ATF1 and HpaBC. Although additional work is needed to reach commercial target levels, the present study opens up a new direction for engineering microbial production of tyrosol acetate and hydroxytyrosol acetate from cheap and readily-available renewable raw materials in the future.

\section{Conclusion}

Tyrosol acetate and hydroxytyrosol acetate have wide applications both as functional food components and as nutraceuticals. There is no report on the bioproductin of them so far. In this study, we demonstrated for the first time the development of microbial cell factories for achieving tyrosol acetate or hydroxytyrosol acetate biosynthesis.

\section{Declarations}

\section{Availability of data and materials}

All data generated or analyzed during this study are included in the article.

\section{Acknowledgements}

Not applicable.

\section{Funding}


This work was financially supported by the National Natural Science Foundation of China (31960216) and National Science Foundation of Jiangxi Province (2018ACB21045 and 20192BCBL23012).

\section{Authors' contributions}

DG and HP conceived and designed the experiments. DG, XF and YS performed the experiments. DG, XF, YS and XL analyzed the data. DG and HP wrote the paper. All authors read and approved the manuscript.

\section{Ethics declarations}

\section{Ethics approval and consent to participate}

Not applicable.

\section{Consent for publication}

Not applicable.

\section{Competing interests}

The authors declare that they have no competing interests.

\section{References}

1. Heim KE, Tagliaferro AR, Bobilya DJ. Flavonoid antioxidants: Chemistry, metabolism and structureactivity relationships. J Nutr Biochem. 2002;13(10):572-84.

2. Cicerale $S$, Lucas LJ, Keast R. Antimicrobial, antioxidant and anti-inflammatory phenolic activities in extra virgin olive oil. Curr Opin Biotechnol. 2012;23(2):129-35.

3. Visioli F, Galli C, Plasmati E, Viappiani S, Hernandez A, Colombo C, et al. Olive Phenol Hydroxytyrosol Prevents Passive Smoking-Induced Oxidative Stress. Circulation. 2000;102(18):2169-71.

4. D'Angelo S, Manna C, Migliardi V, Mazzoni O, Zappia V. Pharmacokinetics and metabolism of hydroxytyrosol, a natural antioxidant from olive oil. Drug Metabolism Disposition. 2001;29(11):1492-8.

5. Clifford M. Practical, Polyphenolics. From Structure to Molecular Recognition and Physiological Action.(Review). Chemistry \& Industry. 1999.

6. Suda I, Oki T, Masuda M, Nishiba Y, Furuta S, Matsugano K, et al. Direct absorption of acylated anthocyanin in purple-fleshed sweet potato into rats. J Agric Food Chem. 2002;50(6):1672-6.

7. Aissa I, Sghair RM, Bouaziz M, Laouini D, Gargouri Y. Synthesis of lipophilic tyrosyl esters derivatives and assessment of their antimicrobial and antileishmania activities. Lipids in Health Disease. 2012;11(1):13.

8. Vlachogianni IC, Fragopoulou E, Kostakis IK, Antonopoulou S. In vitro assessment of antioxidant activity of tyrosol, resveratrol and their acetylated derivatives. Food Chem. 2015;177(15):165-73. 
9. Ahn EY, Jiang Y, Zhang Y, Son E, You S, Kang SW, et al. Cytotoxicity of p-tyrosol and its derivatives may correlate with the inhibition of DNA replication initiation. Oncol Rep. 2008;19(2):527.

10. Rosillo A, Maria, Fernandez-Bolanos, Jose G, Alarcon-de-la-Lastra C, et al. Preventive effects of dietary hydroxytyrosol acetate, an extra virgin olive oil polyphenol in murine collagen-induced arthritis. Molecular Nutrition Food Research. 2015;177:165-73.

11. Tabernero§ M, Sarriá $\infty$ B, Largo§ C, Martínez-López $\infty$ S, Madronał A. Comparative evaluation of the metabolic effects of hydroxytyrosol and its lipophilic derivatives (hydroxytyrosyl acetate and ethyl hydroxytyrosyl ether) in hypercholesterolemic rats. Food Funct. 2014;5(7):1556-63.

12. Feng $Y$, Guangde, Yang, Yushan $X$, et al. The protective effect of hydroxytyrosol acetate against inflammation of vascular endothelial cells partly through the SIRT6-mediated PKM2 signaling pathway. Food function. 2019;10(9):5789-803.

13. Aissa I, Bouaziz M, Ghamgui H, Kamoun A, Miled N, Sayadi S, et al. Optimization of lipase-catalyzed synthesis of acetylated tyrosol by response surface methodology. J Agric Food Chem. 2007;55(25):10298-305.

14. Xue Y, Chen X, Yang C, Chang J, Shen W, Fan Y. Engineering Eschericha coli for Enhanced Tyrosol Production. Journal of Agricultural Food Chemistry. 2017;65:4708-14.

15. Satoh Y, Tajima K, Munekata M, Keasling JD, Lee TS. Engineering of a Tyrosol-Producing Pathway, Utilizing Simple Sugar and the Central Metabolic Tyrosine, in Escherichia coli. Journal of Agricultural Food Chemistry. 2012;60(4):979-84.

16. Haiquan Y, Yuxiang X, Cui W, et al. Modular Engineering of Tyrosol Production in Escherichia coli. J Agric Food Chem. 2019;67:3900-8.

17. Guo D, Zhang, Lihua, Kong S, et al. Metabolic Engineering of Escherichia coli for Production of 2Phenylethanol and 2-Phenylethyl Acetate from Glucose. Journal of Agricultural Food Chemistry. 2018;66:5886-91.

18. Kong S, Zhang J, Li X, Pan H, Guo D. De novo Biosynthesis of Indole-3-ethanol and Indole-3-ethanol acetate in Engineered Escherichia coli. Biochem Eng J. 2019;154:107432.

19. Liu Z, Zong Z, Chen Z, Xu Q, Guo D. De novo Biosynthesis of Antimycobacterial Agent Geranylgeranyl acetate from Glucose. Biochemical Engineering Journal. 2018;142.

20. Bohnenkamp AC, Kruis AJ, Mars AE, Wijffels RH, Weusthuis RA. Multilevel optimisation of anaerobic ethyl acetate production in engineered Escherichia coli. Biotechnol Biofuels. 2020;13:65.

21. Lee JW, Trinh CT. Microbial biosynthesis of lactate esters. Biotechnol Biofuels. 2019;12:226.

22. Li X, Chen Z, Wu Y, Yan Y, Sun X, Yuan Q. Establishing an Artificial Pathway for Efficient Biosynthesis of Hydroxytyrosol. Acs Synthetic Biology. 2017;7:647-54.

23. Gordon MH, Paiva-Martins F, Almeida M. Antioxidant activity of hydroxytyrosol acetate compared with that of other olive oil polyphenols. Journal of Agricultural Food Chemistry. 2001;49(5):2480.

24. González-Correa JA, Navas MD, Muñoz-Marín J, Trujillo M, Cruz JPdl. Effects of Hydroxytyrosol and Hydroxytyrosol Acetate Administration to Rats on Platelet Function Compared to Acetylsalicylic Acid. 
Journal of Agricultural Food Chemistry. 2008;56(17):7872-6.

25. Alcudia F, García Ruano JL, Rodríguez J, Sánchez F. Conformational analysis of acyclic compounds with oxygen-sulphur interactions. Part VI. Some 1-thioderivatives of 2-propanol and its acetates. Can J Chem. 1989;57(18):2426-33.

\section{Figures}

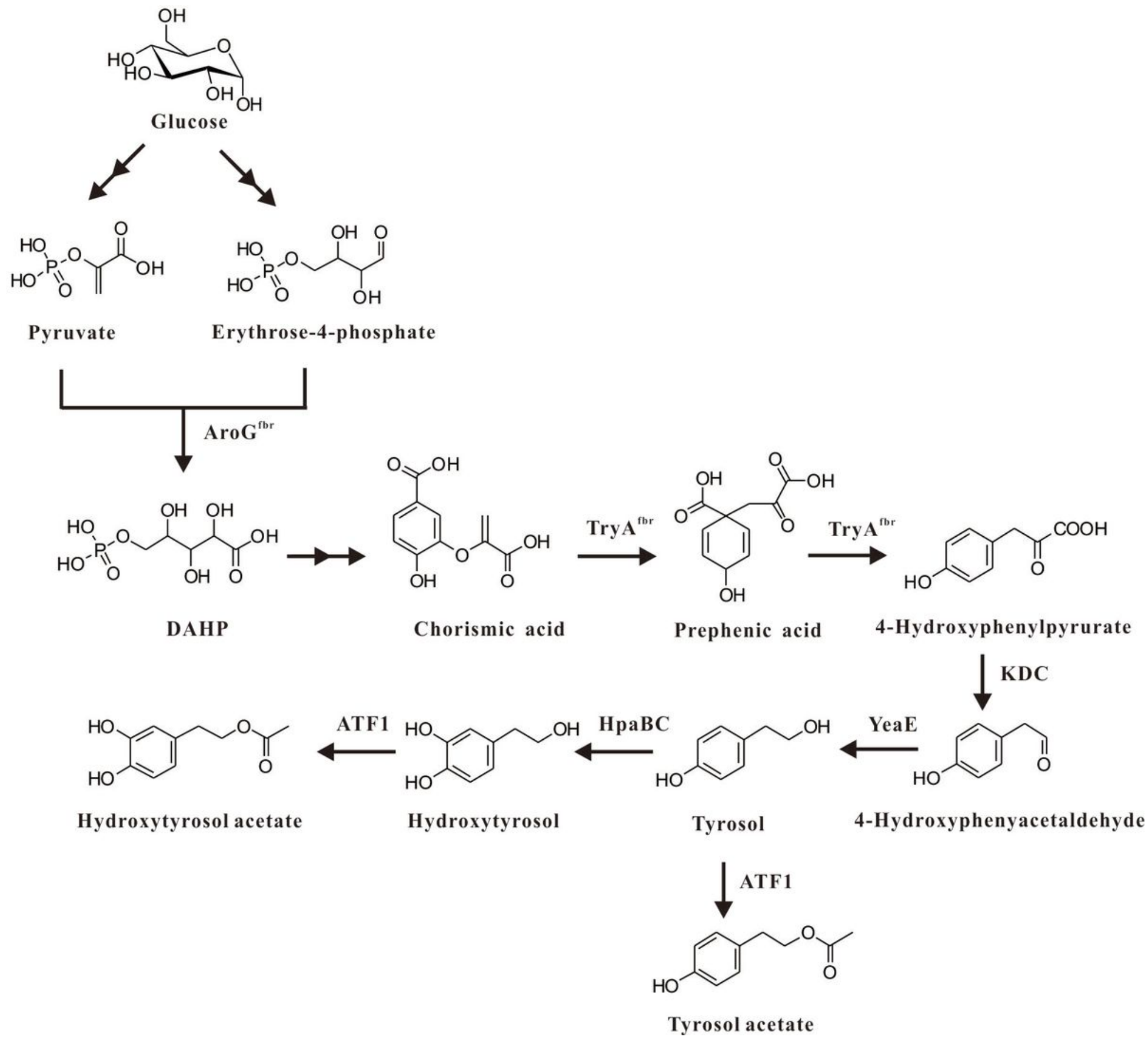

\section{Figure 1}

Engineered pathway for the production of tyrosol acetat and hydroxytyrosol acetate from glucose. 

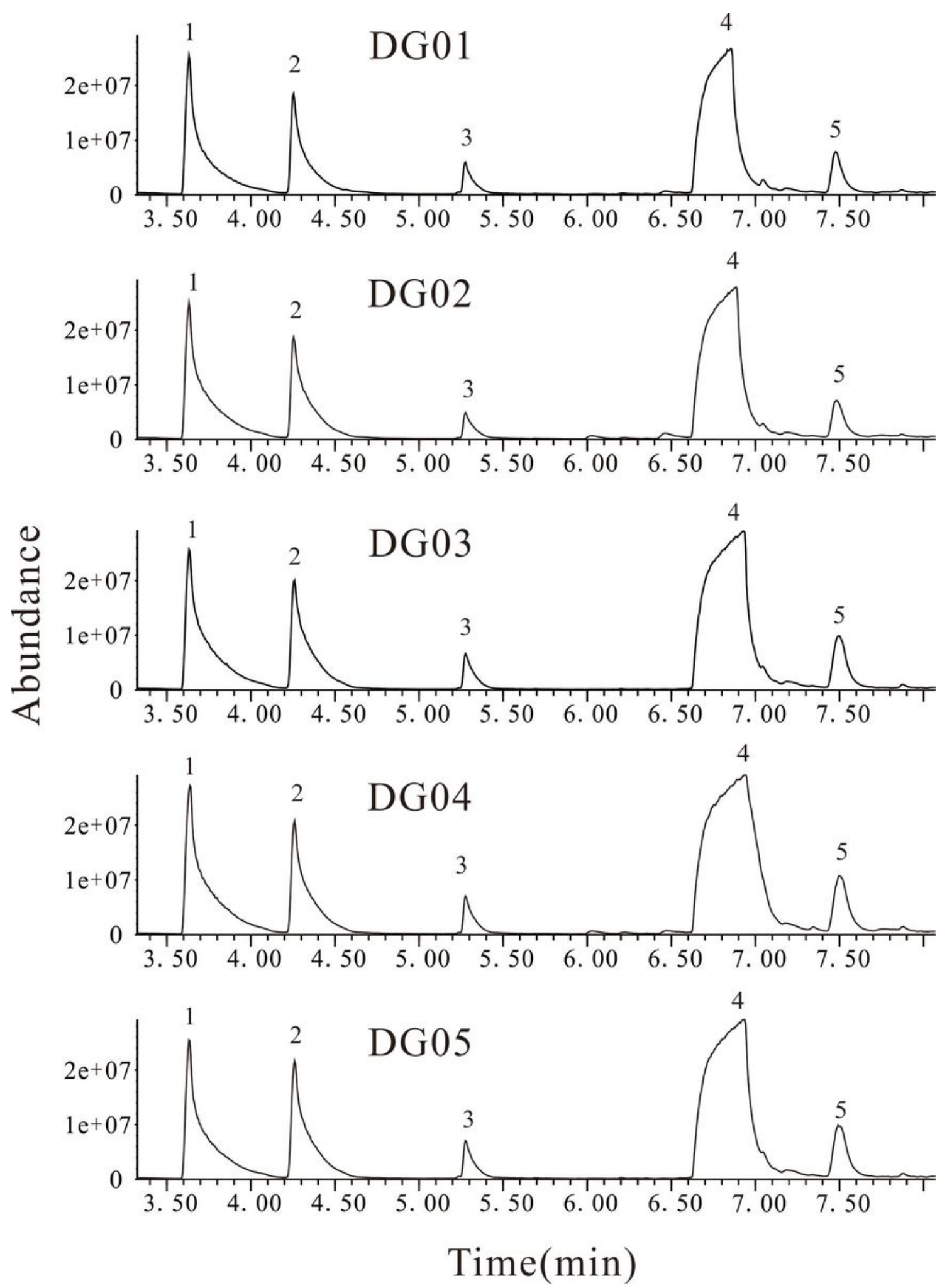

Figure 2

GC/MS analyses of tyrosol in engineered E. coli strains. Identified substances: 1, benzyl alcohol (internal standard); 2 , phenylethanol; 3 , phenylethyl acetate; 4, tyrosol; 5, tyrosol acetate. 


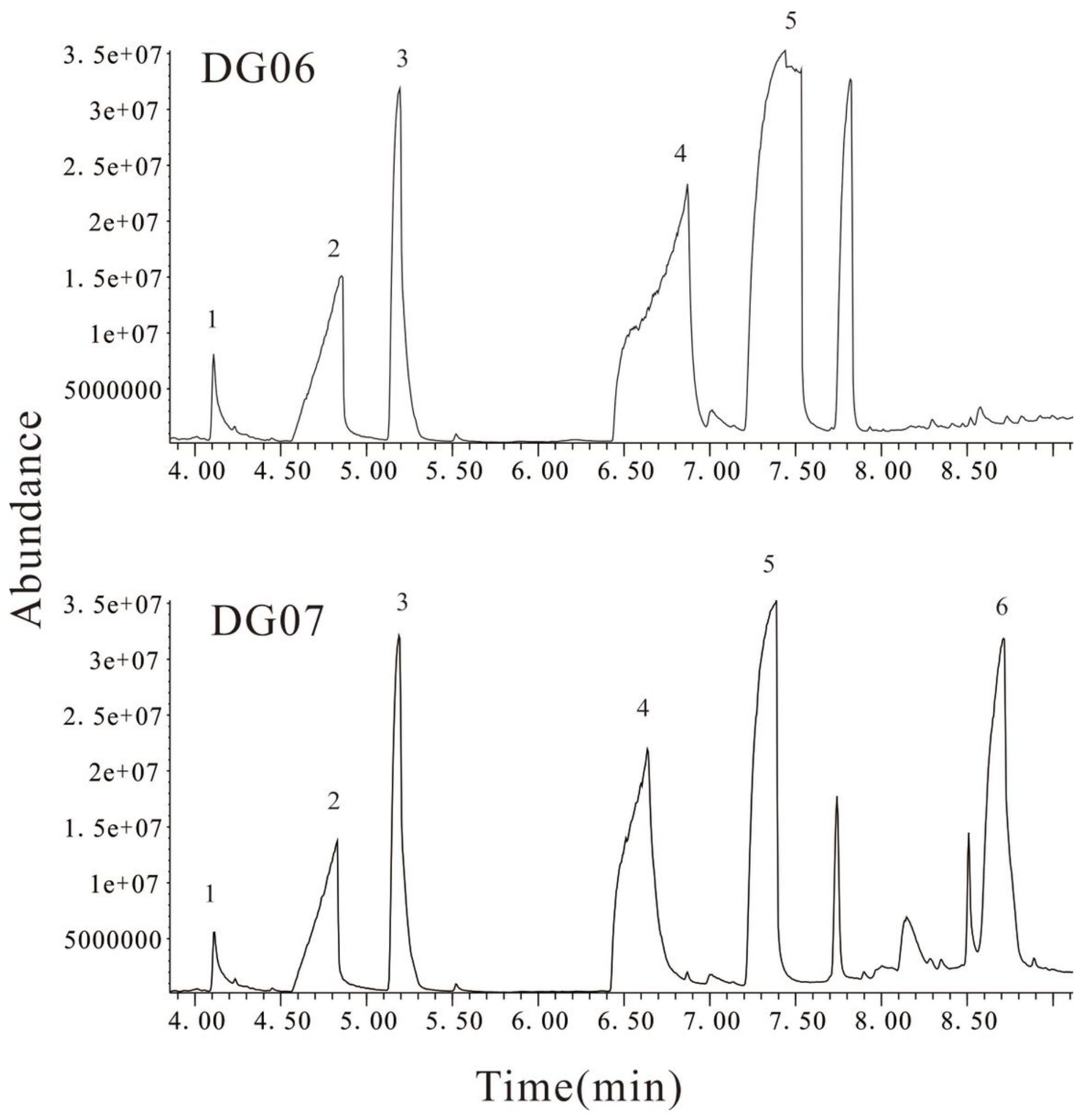

Figure 3

$\mathrm{GC} / \mathrm{MS}$ analyses of tyrosol acetate and hydroxytyrosol acetate in engineered E. coli strains. Identified substances: 1 , phenylethanol; 2, methyl benzoate (internal standard); 3 , phenylethyl acetate; 4, tyrosol; 5 , tyrosol acetate; 6 , hydroxytyrosol acetate. 